\section{FRI0275 HIGH ANGIOPOIETIN-2 LEVELS ASSOCIATE WITH ARTERIAL INFLAMMATION AND LONG-TERM GLUCOCORTICOID REQUIREMENT IN POLYMYALGIA RHEUMATICA}

Yannick van Sleen ${ }^{1}$, Annemieke Boots ${ }^{2}$, Wayel Abdulahad ${ }^{3}$, Johan Bijzet ${ }^{4}$, Maria Sandovici ${ }^{5}$, Kornelis van der Geest ${ }^{6}$, Elisabeth Brouwer ${ }^{6} .{ }^{1}$ University Medical Center Groningen, Rheumatology and Clinical Immunology, Groningen, Netherlands; ${ }^{2}$ University Medical Center Groningen, Rheumatology and Clinical Immunology, Groningen, Netherlands; ${ }^{2}$ University Medical Center Groningen, Rheumatology and Clinical Immunology, Groningen, Netherlands; ${ }^{4}$ University Medical Center Groningen, Rheumatology and Clinical Immunology, Groningen, Netherlands; ${ }^{2}$ University Medical Center Groningen, Rheumatology and Clinical Immunology, Groningen, Netherlands; ${ }^{1}$ University Medical Center Groningen, Rheumatology and Clinical Immunology, Groningen, Netherlands

Background: Polymyalgia rheumatica (PMR) frequently co-occurs with giant cell arteritis (GCA). So far, a simple biomarker for detecting concomitant arterial inflammation in PMR patients is lacking. Furthermore, biomarkers predicting disease course in PMR are awaited.

Objectives: We here investigated the diagnostic and prognostic value of acute-phase markers (ESR, CRP, IL-6, serum amyloid A) and angiogenesis markers (VEGF, soluble Tie2, angiopoietin-1, angiopoietin-2) in isolated PMR and PMR/GCA overlap patients.

Methods: We prospectively included 39 treatment-naive PMR patients, of which 10 patients also showed evidence of large vessel GCA on FDG$\mathrm{PET} / \mathrm{CT}$. Age-matched healthy controls $(\mathrm{N}=32)$ and infection controls $(\mathrm{N}=13)$ were included for comparison. Serum marker levels were measured by ELISA or Luminex. ROC and Kaplan Meier analyses were used to asses diagnostic and prognostic accuracy, respectively.

Results: All acute-phase and angiogenesis markers, except angiopoietin-1, were higher in isolated PMR patients compared to HCs. Angiopoietin-2, ESR and soluble Tie-2 were significantly higher in patients with PMR/ GCA overlap compared to isolated PMR patients. Angiopoeietin-2, but not soluble Tie2, outperformed ESR and CRP in discriminating patients with and without overlapping GCA (AUC 0.90). Moreover, high angiopoietin-2 levels were associated with long-term glucocorticoid requirement.

Conclusion: Assessment of angiopoietin-2 at baseline may assist diagnosis of concomitant vasculitis in PMR. Moreover, high levels of angiopoietin-2 were associated with a unfavorable disease course in isolated PMR patients. These findings imply that angiopoietin-2 could be an interesting diagnostic and prognostic biomarker in PMR.

Disclosure of Interests: Yannick van Sleen: None declared, Annemieke Boots Grant/research support from: grant from MSD in 2010-2015, Consultant for: I was a consultant for Grunenthal Germany 2016-2017, Employee of: I was an employee of Organon, Shering-Plough and MSD from 1991-2011, Wayel Abdulahad: None declared, Johan Bijzet: None declared, Maria Sandovici: None declared, Kornelis van der Geest: None declared, Elisabeth Brouwer Speakers bureau: Dr. Brouwer as an employee of the UMCG received speaker fees and consulting fees from Roche which were paid to the UMCG DOI: 10.1136/annrheumdis-2019-eular.5394

\section{FRI0276 TIME OF DISEASE EVOLUTION AND EFFICACY OF TOCILIZUMAB IN GIANT CELL ARTERITIS}

Monica Calderón-Goercke, J. Loricera, D. Prieto-Peña, Vicente Aldasoro, Santos Castañeda, Ignacio Villa-Blanco, Alicia Humbría, Clara Moriano, Susana Romero-Yuste, J. Narváez, Catalina Gomez-Arango, Eva Perez-Pampín, Rafael Melero, Elena Becerra-Fernández, Marcelino Revenga, Noelia AlvarezRivas, Carles Galisteo, Francisca Sivera, Alejandro Olive, María Álvarez del Buergo, Luisa Marena Rojas, Carlos Fernández-López, Francisco Navarro, Enrique Raya, Eva Galindez, Beatriz Arca, Roser Solans-Laqué, Arantxa Conesa, Cristina Hidalgo, Carlos Vázquez, Jose Andrés Román-Ivorra, Pau Lluch, Sara Manrique Arija, Paloma Vela-Casasempere, Eugenio de Miguel, Carmen TorresMartín, Juan Carlos Nieto, Carmen Ordas-Calvo, Eva Salgado-Pérez, Cristina Luna-Gomez, J. Francisco. Toyos Sáenz de Miera, Nagore FernándezLlanio, Antonio García, Carmen Larena, Natalia Palmou-Fontana, Vanesa CalvoRío, Carmen González-Vela, Alfonso Corrales, María Varela-García, Elena Aurrecoechea, Raquel Dos-Santos, Ángel García-Manzanares, Norberto Ortego, Sabela Fernández, Francisco Ortiz-Sanjuán, Montserrat Corteguera, J. Luis Hernández, Miguel A. González-Gay, Ricardo Blanco. Rheumatology, Internal Medicine and Pathology Units, Santander, Navarra, Madrid, Torrelavega, León, Pontevedra, Barcelona, Mondragón, Santiago de Compostela, Vigo, Alicante, Lugo, Badalona, Palencia, Alcázar de San Juan, A Coruña, Granada, Bilbao, Avilés, Castellón, Salamanca, Zaragoza, Valencia, Menorca, Málaga, Ávila, Gijón, Ourense, Tenerife, Sevilla, Lérida, Spain

Background: Tocilizumab (TCZ) has proved to be effective in the management of Giant Cell Arteritis (GCA). Based on results of the GiACTA trial, it has been approved by the FDA and the European Commission for GCA treatment. Nevertheless, almost half of the GiACTA trial patients presented a short time evolution disease.

Objectives: Our aim was to evaluate the efficacy of TCZ according the time of disease evolution.

Methods: Retrospective, multicenter study of 134 patients with GCA in treatment with TCZ. A comparative study between two groups according to the time from disease diagnosis and TCZ onset was performed.

Results: Our study included 134 GCA patients. TABLE 1 summarizes a comparative study between: a) $\leq 6$ months of disease evolution, and b) $>6$ months of disease evolution. Non-significant difference in baseline characteristics was found. In terms of visual involvement, we observed more patients affected in the first group $(\leq 6$ months) $(\mathrm{p}=0.30)$. Analyzing clinical improvement non-significant difference was seen during follow-up. At TCZ onset, in the group of $\leq 6$ months of evolution, the prednisone dose was higher with a mean dose of $31.3 \pm 16.5 \mathrm{mg} / \mathrm{d}$ vs $17.7 \pm 14.2 \mathrm{mg} /$ $d(p<0.001)$, however, a similar reduction of corticosteroids was achieved in both groups after 6 months of follow-up. The incidence of adverse events and severe infections was similar in both groups $(p=0.132$ and $\mathrm{p}=0.672$ respectively).

Conclusion: In this retrospective analysis, our results support the previously reported efficacy and safety profile of TCZ. We can conclude that TCZ can be used in GCA independently the time of disease evolution.

\section{REFERENCES:}

[1] Calderón-Goercke M. Tocilizumab in giant cell arteritis. Observational, open-label multicenter study of 134 patients in clinical practice. Semin Arthritis Rheum. 2019 Jan 5. pii: S0049-0172(18)30571-7. Doi: 10.1016/j. semarthrit.2019.01.003. [Epub ahead of print]

[2] Salvarani C, Magnani L, Catanoso M, Pipitone N, Versari A, Dardani L, et al. Tocilizumab: a novel therapy for patients with large-vessel vasculitis. Rheumatolgy (Oxford). 2012; 51:151-6

[3] Stone JH, Tuckwell K, Dimonaco S, Klearman M, Aringer M, Blockmans D, et al. Trial of Tocilizumab in Giant-Cell Arteritis. N Engl J Med. 2017; 377:317-28.

\begin{tabular}{|c|c|c|c|}
\hline & $\begin{array}{c}\text { GCA } \leq 6 \text { MESES } \\
(n=44)\end{array}$ & $\begin{array}{c}\text { GCA }>\text { 6 MESES } \\
(n=90)\end{array}$ & $\mathrm{p}$ \\
\hline CORTICOSTEROIDS AT TCZ ONSET & & & \\
\hline Prednisone dose, mg/d mean (SD) & $31.3 \pm 16.5$ & $17.7 \pm 14.2$ & $<0.001$ \\
\hline EFFICACY AND SAFETY AFTER TCZ & & & \\
\hline $\begin{array}{l}\text { Prolonged remission } n(\%) \\
\text { Montt } 6 \\
\text { Month } 12\end{array}$ & $\begin{array}{l}16(57.1) \\
11(64.7)\end{array}$ & $\begin{array}{l}42(58.3) \\
35(67.3)\end{array}$ & $\begin{array}{l}0.272 \\
0.843\end{array}$ \\
\hline $\begin{array}{l}\text { Month 24 } \\
\text { Relapses } \mathrm{n}(\%) \\
\text { Month } 1 \\
\text { Month } 3 \\
\text { Month } 6 \\
\text { Month } 12 \\
\text { Month } 24\end{array}$ & $\begin{array}{l}6(66.7) \\
2(4.9) \\
4(10.8) \\
1(3.6) \\
2(11.8) \\
1(11.1)\end{array}$ & $\begin{array}{l}21(67.7) \\
2(2.3) \\
3(3.5) \\
4(5.6) \\
7(13.5) \\
6(19.4)\end{array}$ & $\begin{array}{l}0.952 \\
0.593 \\
0.197 \\
1.000 \\
0.857 \\
0.567\end{array}$ \\
\hline $\begin{array}{l}\text { CORTICOSTEROIDS SPARING EFFECTS, median [IQR] } \\
\text { Month } 1 \\
\text { Month } 3 \\
\text { Month } 6 \\
\text { Month } 12 \\
\text { Month } 24\end{array}$ & $\begin{array}{c}18.8[10-30] \\
12.5[7.5-20] \\
5[4.4-10.6] \\
5[0-5] \\
0[0-4.4]\end{array}$ & $\begin{array}{l}0[19.4] \\
10[5-15] \\
7.5[5-10] \\
5[2.5-7.5] \\
2.5[0-5] \\
0[0-5]\end{array}$ & $\begin{array}{l}<.001 \\
<0.001 \\
<0.001 \\
0.202 \\
0.335 \\
0.724\end{array}$ \\
\hline SIDE EFFECTS, n (\%) & & & \\
\hline Relevant adverse events & $14(31.8)$ & $18(24)$ & 0.132 \\
\hline
\end{tabular}

Disclosure of Interests: Monica Calderón-Goercke: None declared, J. Loricera: None declared, D. Prieto-Peña: None declared, Vicente Aldasoro: None declared, Santos Castañeda Consultant for: Amgen, BMS, Pfizer, Lilly, MSD, Roche, Sanofi, UCB, Ignacio Villa-Blanco: None declared, Alicia Humbría: None declared, Clara Moriano: None declared, Susana Romero-Yuste: None declared, J. Narváez Consultant for: Bristol-Myers Squibb, Catalina Gomez-Arango: None declared, Eva Perez-Pampín: None declared, Rafael Melero: None declared, Elena Becerra-Fernández: None declared, Marcelino Revenga: None declared, Noelia Alvarez-Rivas: None declared, Carles Galisteo: None declared, Francisca Sivera: None declared, Alejandro Olive: None declared, María Álvarez del Buergo: None declared, Luisa Marena Rojas: None declared, Carlos FernándezLópez: None declared, Francisco Navarro: None declared, Enrique Raya: None declared, Eva Galindez: None declared, Beatriz Arca: None declared, Roser Solans-Laqué: None declared, Arantxa Conesa: None declared, Cristina Hidalgo: None declared, Carlos Vázquez: None declared, Jose Andrés Román-lvorra: None declared, Pau Lluch: None declared, Sara Manrique Arija Speakers bureau: ABBvie, MSD, Janssen, Lilly, Roche, Pfyzer, Novartis., Paloma Vela-Casasempere Grant/research support from: UCB, Abbvie, Pfizer, Roche, Bristol-Myer-Squibb (another research, not BIOBADASER related), Consultant for: UCB, Lilly, Pfizer, Roche, Bristol-Myer-Squibb, Speakers bureau: Roche, UCB, MSD, Pfizer, GSK, BMS, Lilly, Eugenio de Miguel: None declared, Carmen Torres-Martín: None declared, Juan Carlos Nieto: None declared, Carmen OrdasCalvo: None declared, Eva Salgado-Pérez: None declared, Cristina Luna- 